\title{
Antibody-inspired affinity measurements of fluorescein-linked small molecule ligands
}

\author{
Marco Catalano ${ }^{1}$, Sebastian Oehler ${ }^{1}$, Luca Prati $^{2}$, Nicholas Favalli ${ }^{1}$, Gabriele Bassi ${ }^{1}$, Jörg Scheuermann ${ }^{1}$, Dario \\ Neri $^{1}$ \\ ${ }^{1}$ Department of Chemistry and Applied Biosciences, Swiss Federal Institute of Technology (ETH Zürich), Vladimir-Prelog-Weg 4, \\ CH-8093 Zurich (Switzerland) \\ ${ }^{2}$ Philochem AG, Libernstrasse 3, 8112 Otelfingen (Switzerland)
}

\section{KEYWORDS: Affinity measurement, ELISA, SPR, Binding Assays, DNA-Encoded Library}

\begin{abstract}
The availability of reliable methods for the characterization of the binding of small molecule ligands to protein targets is crucially important for Drug Discovery. We have adapted a method, routinely used for the characterization of monoclonal antibodies (Enzyme-linked immunosorbent assay, or "ELISA"), to small molecule ligands, using fluorescein conjugates and anti-fluorescein antibodies as detection reagents. The new small molecule-ELISA methodology was tested using a panel of binders specific to carbonic anhydrase II, with dissociation constants ranging between $6 \mu \mathrm{M}$ and $14 \mathrm{nM}$. An excellent agreement was found between ELISA measurements and fluorescence polarization results. The methodology was also extended to BIAcore measurements and implemented for ligands coupled to oligonucleotides. Small molecule-ELISA procedures are particularly useful in the context of DNA-
\end{abstract}

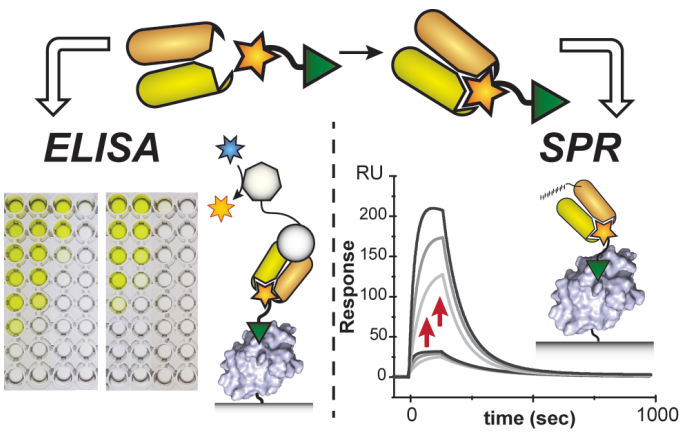
encoded libraries, for which hit validation procedures need to be performed on dozens of candidate molecules and hit compounds can be conveniently resynthesized on DNA.

Small molecule therapeutic agents are organic compounds (typically smaller than $500 \mathrm{Da}$ ), which confer a benefit to patients thanks to their interaction with cognate protein targets. The discovery of binding molecules and the quantitative characterization of their protein binding properties represents a fundamental step in the drug discovery process. ${ }^{1-4}$

A number of methodologies exist for the determination of the dissociation constants between small molecules and protein targets, but each of them has some limitation. For example, enzyme inhibition assays can be extremely useful for the discovery of small molecule inhibitors, but not all pharmaceutical targets are enzymes. ${ }^{5,6}$ Isothermal titration calorimetry (ITC) allows the measurement of dissociation constants and other thermodynamic parameters (e.g., change in enthalpy and in entropy) in solution, but the method requires substantial amounts of protein and the precise matching of solvents. ${ }^{7}$ Sample limitations apply also for NMR methodologies which commonly require relatively large amounts of isotopically labelled protein (i.e., ca. $30 \mathrm{nmol}$ per compound) and which preferably work with proteins smaller than $30-40 \mathrm{kDa}{ }^{8}$ Surface plasmon resonance (SPR) is frequently used for the real-time analysis of biomolecular interactions. In a typical setup, binding events are detected between a protein immobilized on a sensor surface and a ligand, reaching the sensor chip through a flow cell. ${ }^{9}$ Since SPR signal is related to the mass change at the sensor surface, interactions between small molecules and protein may suffer from insufficient sensitivity. ${ }^{10}$ Large concentrations of small ligands may require the use of organic solvents (e.g., DMSO), which are not always compatible with SPR applications. ${ }^{11}$ In view of these considerations, it would be desirable to develop analytical methods for the characterization of the interaction between small molecules ligands and their cognate protein target, while using minute amounts of reagents in aqueous environment.
The need for efficient methods for the study of protein-ligand interactions has become more acute thanks to advances in DNAencoded library (DEL) technology. DELs are based on the covalent attachment of small organic compounds at the extremities of DNA strands. ${ }^{12}$ The synthesis of small molecule compounds on DNA is typically performed in a combinatorial fashion and each combination of building-blocks is individually identified by specific DNAsequences acting as amplifiable barcodes. ${ }^{13,14}$ DEL-based drug discovery campaigns commonly result in the identification of hundreds of candidates, that need to be re-synthesized and validated.

The hit validation problems associated with the implementation of DEL technology are reminiscent of discoveries made in the early '70s, when enzyme-linked immunosorbent assays (ELISA) allowed the facile characterization of hundreds of different binding specificities, using minute amounts of samples. ${ }^{15,16}$ In ELISA procedures, a binding component (e.g., a protein antigen) is coated on plastic wells and can be exposed to different concentrations of a binding partner (e.g., an antibody), using a suitable enzyme-based detection system (e.g., through the use of enzyme-linked secondary antibody reagents and chromogenic substrates).

In this work, we adapted the ELISA methodology in order to detect small molecule-protein interactions. We made use of fluorescein conjugates of small organic ligands, which could simultaneously interact with the target protein of interest and with suitable high-affinity anti-fluorescein antibody reagents. The methodology was implemented in ELISA format and in other conceptually related systems (e.g., BIAcore), using carbonic anhydrase II (CAII) as target antigen. Two recombinant antibody formats (i.e., scFv and IgG) were used, in order to provide a kinetically stable complexation of the fluorescein moiety with monomeric or homodimeric interaction partners. Finally, we used anti-fluorescein antibody reagents for the 
ELISA characterization of ligands coupled to fluoresceinated DNAstructures, thus paving the way to hit validation procedures for ligands isolated from DEL libraries.

\section{EXPERIMENTAL SECTION}

Reagents. Starting materials, solvents, and reagents, unless otherwise noted, were used as supplied by Fisher Chemicals, Merck, VWR or Sigma-Aldrich in HPLC or analytical grade. All other reagents (carboxylic acids, isothiocyanates) were purchased from several commercial suppliers including Enamine, ABCR and Sigma Aldrich. Oligonucleotides were purchased from LGC Biosearch Technologies. Power- $\mathrm{CHO}$ and Pro-CHO medium for cell culturing were purchased from Lonza and supplied with $8 \mathrm{M}$ Ultraglutamine (Gibco), 4 MHT (Gibco), and 1\% antibiotic-antimycotic (Gibco). CAII from bovine erythrocytes was purchased from Sigma-Aldrich (Cat. n. C2624). Monoclonal Anti-His-HRP, streptavidin-HRP and proteinA-HRP protein conjugates were purchased from BioLegend. A pre-made solution of 3,3',5,5'-tetramethylbenzidine (TMB) was purchased from Sigma-Aldrich (Cat. n. T4444). Tween 20 was purchased from Sigma-Aldrich (P6585). SPR measurements were performed on a BIAcore S200 (Cytiva, formerly GE Healthcare Life Sciences). CM5 BIAcore microsensor chips and other BIAcore associated reagents (e.g., HPS-EP buffer, vials, etc.) were purchased from Cytiva.

Cloning of anti-FITC antibodies. A synthetic gene, coding for a previously-described anti-fluorescein antibody in $\mathrm{scFv}$ format $(4 \mathrm{~m} 5.3)^{17,18}$ served as template for the subcloning and expression of recombinant antibody products in $\mathrm{scFv}$ and $\mathrm{IgG}$ format, using published procedures. ${ }^{19}$ The complete amino acid sequence of the two antibodies is shown in Supplementary Figures S4-S5, together with a schematic representation of the expression plasmids.

Protein expression and purification. Transient gene expression was performed according to previously published methodologies in Chinese hamster ovary (CHO-S) cells. ${ }^{20} \mathrm{CHO}-\mathrm{S}$ cells were cultured according to supplier's protocol. The IgG antibody was purified from the supernatant by protein-A affinity chromatography, eluted with $0.1 \mathrm{M}$ glycine $\mathrm{pH} 3$ and subsequently dialyzed into 500-fold volume of PBS pH 7.4 overnight. The scFv antibody was purified from the supernatant by Ni-NTA affinity chromatography resin (COHISR-RO Roche), eluted with $250 \mathrm{mM}$ Imidazole $\mathrm{pH} 7.8$ and subsequently dialyzed into 500-fold volume of $\mathrm{PBS} \mathrm{pH}$ 7.4. For quality control, the proteins were characterized by size exclusion chromatography on a Äkta Pure FPLC system (GE Healthcare) with a Superdex S200 10/300 increase column (GE Healthcare), by SDS-PAGE and by mass spectrometry.

Synthesis. Small molecule ligands and fluoresceinated on DNA compounds were produced according to already described procedures. $^{21,22}$

SPR evaluation of Fluorescein binding. A microsensor chip CM5 was let equilibrate at room temperature, washed with milliQwater and dried over nitrogen before usage. The chip was equilibrated in a BIAcore series $S$ instrument with HBS-EP buffer until stable signal was reached. The scFv and the IgG were covalently coated on the CM5 chip following software-templated procedures. For immobilization, proteins were diluted at $1 \mu \mathrm{M}$ concentration in acetate buffer $\mathrm{pH}$ 5. Coating densities were $3078 \mathrm{RU}$ and $6494 \mathrm{RU}$ for the $\mathrm{scFv}$ and the IgG, respectively. Immediately after the coating step, the microsensor was let equilibrate in PBS pH 7.4 (Gibco) supplemented with $0.005 \%$ Tween 20 . At reached signal stability, a solution of Fluorescein- $\mathrm{PEG}_{2}-\mathrm{NH}_{2}(\mathrm{NH} 2) 8 \mathrm{nM}$ was flown on the chip surface (association time $120 \mathrm{sec}$, dissociation time $600 \mathrm{sec}$, flow $30 \mu \mathrm{L} / \mathrm{min}$ ).

Protein Biotinylation. Carbonic anhydrase 2 from bovine erythrocytes (C2624, Sigma-Aldrich) was suspended in 5\% DMSO carbonate buffer $(0.1 \mathrm{M}, \mathrm{pH} 9)$ in presence of 2.5 equivalents of EZLink NHS-LC-Biotin (ThermoFisher). After $1 \mathrm{~h}$ of gentle mixing, the solution was purified by disposable PD 10 desalting column (GE Healthcare) in buffer PBS pH 7.4 (Gibco). A random distribution of lysine modifications was obtained with average protein to biotin ratio of ca. 2 [Supplementary Figure 1].

Small molecule ELISA. Biotinylated carbonic anhydrase 2 (bCAII) was incubated for 2 hours at room temperature on StreptaWell (Roche). The supernatant was removed and $200 \mu \mathrm{L}$ of a $4 \%$ solution of Milk powder in PBS (M-PBS) was used as blocking agent for 30-45 minutes. Wells were then washed three times with $200 \mu \mathrm{L}$ PBS and dried. $100 \mu \mathrm{L}$ of a pre-made serial dilution of the fluorescein-ligand conjugate was added and incubated for 45 minutes in the dark. Carefully, the supernatant was removed and the wells were quickly washed twice with $200 \mu \mathrm{L}$ PBS. $100 \mu \mathrm{L}$ of either IgG (200 $\mathrm{nM})$ or $\mathrm{scFv}(400 \mathrm{nM})$ in $2 \% \mathrm{M}$-PBS were added and incubated for 45 minutes. The supernatant was removed and wells washed three times with $200 \mu \mathrm{L}$ PBS. $100 \mu \mathrm{L}$ of a solution of 1:1000 diluted enzyme-linked protein (i.e., proteinA-HRP or anti-His-HRP) in $2 \%$ M-PBS was added and incubated for 45 minutes in the dark. The supernatant was removed and wells washed three times with $200 \mu \mathrm{L}$ of a $0.1 \%$ solution of Tween 20 in PBS and three times with PBS. Finally, $60 \mu \mathrm{L}$ of 3,3',5,5'-tetramethylbenzidine (TMB) was added to each well and left for 10 to 50 seconds at room temperature developing in the dark until formation of a visible intense blue. The reaction was then quenched upon addition of $30 \mu \mathrm{L}$ of $1 \mathrm{M} \mathrm{H}_{2} \mathrm{SO}_{4}$. The absorbance at $450 \mathrm{~nm}$ and $620 \mathrm{~nm}$ was measured with Spectra Max Paradigm multimode plate reader (Molecular Devices) and values were fitted using Graphpad Prism software. The method is compatible with different buffers and with the addition of solvents (e.g., DMSO). However, the presence of reducing agents in the buffer mixture might significantly impair the final oxidative step (data not shown).

Fluorescence polarization. In a total volume of $30 \mu \mathrm{L}$, a serial dilution of CAII in PBS pH 7.4 was incubated with $12.5 \mathrm{nM}$ fluoresceinated small molecule ligands. Dilutions were performed in non-binding 384-well black microplates (Greiner Bio-one) and fluorescence anisotropy was measured on a Spectra Max Paradigm multimode plate reader (Molecular Devices). Anisotropy values were fitted using Graphpad Prism software.

On DNA ELISA. Compounds displayed on a single stranded 5'C6-amino 12-mer DNA were incubated $5 \mathrm{~min}$ at $65^{\circ} \mathrm{C}$ in presence of a stoichiometric amount of a complementary 5'-FITC 12-mer LNA strand. The mixture was let to cool down at room temperature for at least $20 \mathrm{~min}$ to ensure the annealing. Serial dilutions of the heteroduplex were performed in PBS prior to the addition in the CAII coated wells (duplicates). The rest of the experiment was performed in full analogy with the small molecule ELISA using $200 \mathrm{nM} \mathrm{IgG}$ as primary antibody.

Inverse-ELISA. Nunc MaxiSorp ${ }^{\mathrm{TM}}$ ELISA stripes (ThermoFisher) were incubated overnight with $100 \mathrm{nM}$ IgG in PBS at $4^{\circ} \mathrm{C}$. The supernatant was removed and $200 \mu \mathrm{L}$ of a $4 \%$ solution of 
Milk powder in PBS (M-PBS) was used as blocking agent for 45 minutes. Wells were then washed three times with $200 \mu \mathrm{L}$ PBS and dried. $50 \mu \mathrm{L}$ of a pre-made $10 \mathrm{nM}$ solution in PBS of DNA:LNAFITC heteroduplex was added in each well (duplicate for each binder) and incubated for $45 \mathrm{~min}$ in the dark. The supernatant was carefully removed and wells were washed twice with $200 \mu \mathrm{L}$ PBS and dried. Wells were incubated with $100 \mu \mathrm{L}$ of a serial dilution of bCAII in M-PBS $2 \%$ for 45 minutes. The supernatant was carefully removed and wells were washed twice with $200 \mu \mathrm{L}$ PBS. $100 \mu \mathrm{L}$ of a solution of 1:1000 diluted streptavidin-HRP in 2\% M-PBS was added in each well and incubated for 45 minutes in the dark. The rest of the experiment was performed in full analogy with the small molecule ELISA (reaction time 5-10 minutes).

Multi-Cycle SPR. Series S Sensor Chip CM5 (GE Healthcare) was equilibrated in HBS-EP Buffer (GE Healthcare) at $30 \mu \mathrm{L} / \mathrm{min}$ in a BIAcore S200. All experiments were carried out at $25^{\circ} \mathrm{C}$. Following template-aided procedure (i.e., amine coupling), $1 \mu \mathrm{M}$ bovine CAII dissolved in buffer acetate $\mathrm{pH} 5.0$ and immobilized on the CM5 Chip following the EDC/NHS protocol. The reached immobilized target level was 1145 RU. The reference flow-cell was blankimmobilized following the templated procedure. The microsensor was then equilibrated in PBS buffer $\mathrm{pH} 7.4$ (Gibco) with $0.05 \%$ Tween 20 (0.05\% PBS-T). Separately, fluorescein-conjugated compounds were dissolved and filtered at millimolar concentration in

PBS without the addition of organic solvents. Concentration was evaluated from the maximum intensity of absorbance of fluorescein (i.e., $495 \mathrm{~nm}$ ) using extinction coefficient of $73000 \mathrm{M}^{-1} \mathrm{~cm}^{-1} .100 \mu \mathrm{M}$ stock solutions in $0.05 \%$ PBS-T were prepared for later uses. Biacore measurements were performed starting from a 1:1 serial dilution of each compound in $0.05 \%$ PBS-T. An identical serial dilution was prepared with fluorescein-linked compounds pre-incubated with a stoichiometric amount of the anti-fluorescein $\mathrm{scFv}$ dissolved in $0.05 \%$ PBS-T. The measurements were performed following the templated multi-cycle analysis (association time 140 seconds, dissociation time 2400 seconds, flow $30 \mu \mathrm{L} / \mathrm{min}$ ). Kinetic fitting was performed using the BIAcore S200 evaluation software (Version 1.0) using a 1:1 binding mode. Raw data of each measurement are presented in Supplementary Figures S7-S12.

\section{RESULTS}

We used bovine carbonic anhydrase 2 (CAII) and cognate aromatic sulfonamide ligands as model system for the implementation of small molecule ELISA procedures [Figure 1A]. Three
A

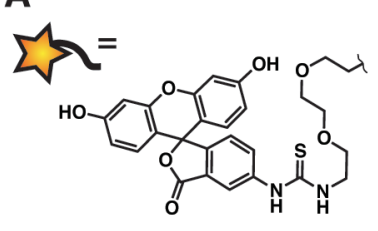

Fluorescein-PEG ${ }^{-}$

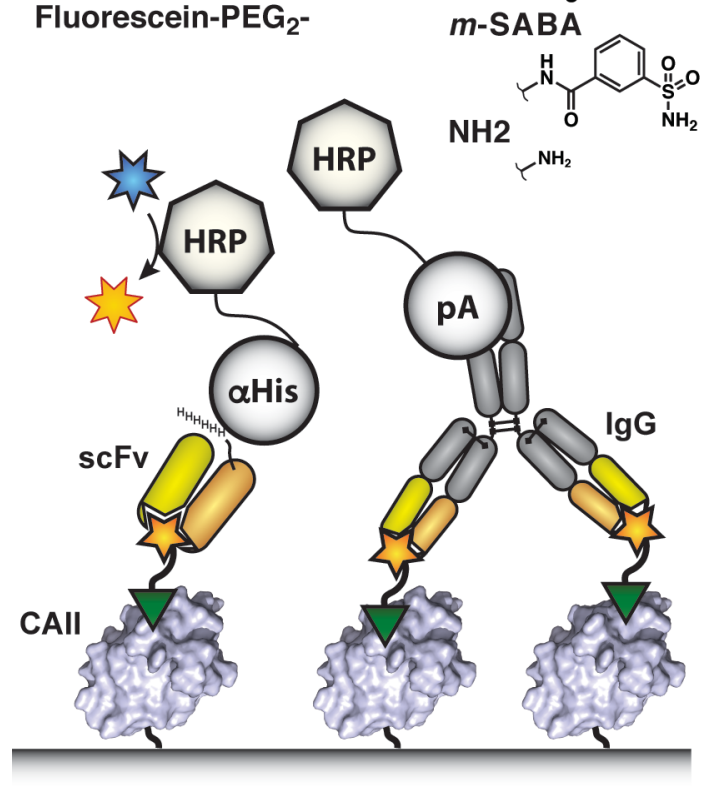

B

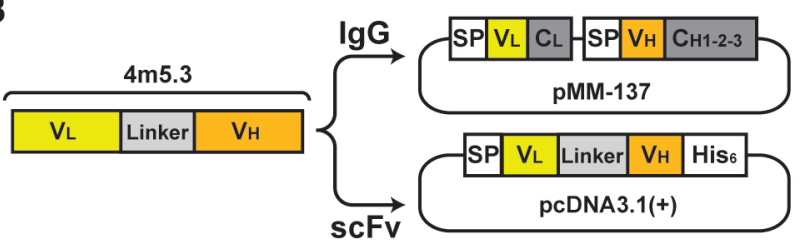

C
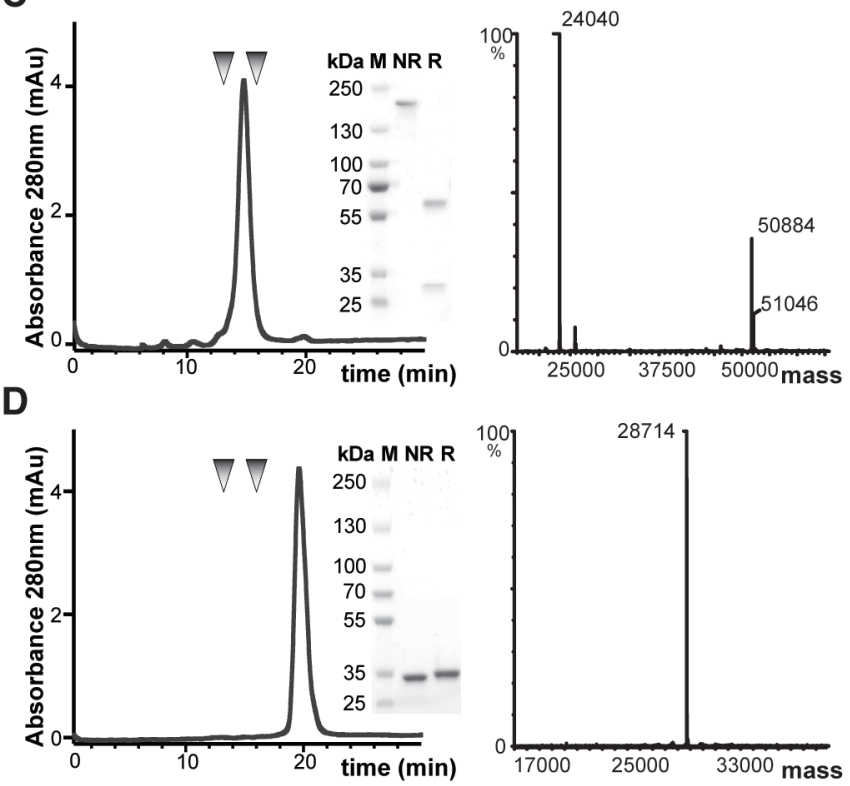

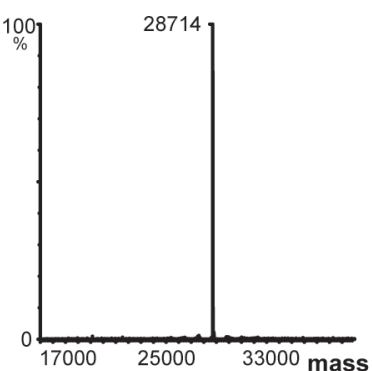

Figure 1. Two different variants of a high-affinity antibody against fluorescein were used and characterized for ELISA measurements of small molecule binders. A) Three fluorescein-linked binders (AAZ, SABA, and m-SABA) with different affinities for carbonic anhydrase 2 (CAII) were investigated in ELISA assays. An analogue free-amino compound was used as negative control (NH2). B) the variable light (VL) and the variable heavy (VH) chains of the parental $4 \mathrm{~m} 5.3$ sequence was cloned within a human DP47 immunoglobulin 1 (IgG) scaffold and in single-chain variable fragment (scFv) format with an additional His-tag sequence. C) Quality control analysis of the IgG was performed via size-exclusion chromatography, SDS-PAGE gel-electrophoresis, MS analysis and used without further purification. D) Quality control analysis of the scFv was performed by size-exclusion chromatography, SDS-PAGE gel-electrophoresis, and MS analysis. In this case, the protein had been furtherly purified with a preparative size-exclusion chromatography in order to reduce the amount of small-aggregates from the initial batch [Supplementary Figure S2].

${ }^{*} \mathrm{M}=$ molecular weight size marker; $\mathrm{NR}=$ non-reducing conditions; $\mathrm{R}=$ reducing conditions; Size-exclusion chromatography standard peaks are obtained with bovine serum albumin $(\mathrm{MW}=66.43 \mathrm{kDa})$ and lysozyme $(\mathrm{MW}=14.30 \mathrm{kDa})$ [Supplementary Figure S3]. 


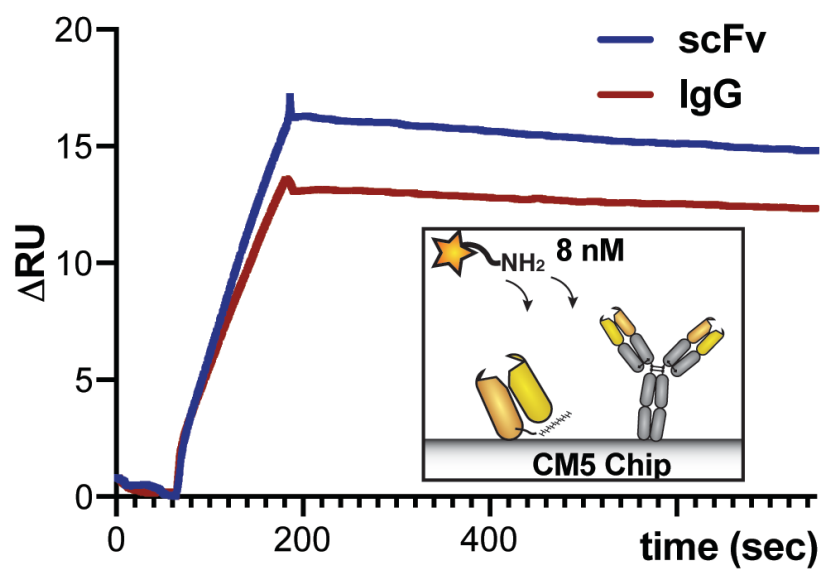

Figure 2. Evaluation of the fluorescein binding capacity of purified antibodies (i.e., IgG and scFv) through SPR analysis. A single $8 \mathrm{nM}$ injection of a fluorescein-conjugate (NH2) was flown on the immobilized chip surface with scFv and IgG (immobilization levels were 3078 RU and 6494 RU, respectively).

different small molecule ligands, based on acetazolamide (AAZ), 4sulfamoylbenzoic amide (SABA) and 3-sulfamoylbenzoic amide ( $m$-SABA), were coupled to fluorescein, in order to generate bifunctional conjugates of different affinity to CAII $\left(\mathrm{K}_{\mathrm{D}}=12.1 \mathrm{nM}, 151\right.$ $\mathrm{nM}$ and $8.6 \mu \mathrm{M}$, respectively). ${ }^{23,24}$ A diamino- $\mathrm{PEG}_{2}$ linker was employed as spacer between the ligand and the fluorophore [Figure 1A]. Coating of plastic wells with a target protein of interest (e.g., CAII) should enable the capture of fluoresceinated binding molecules, followed by an antibody-based detection of the fluorescein moiety [Figure 1A]. In order to assess the impact of antibody valence on ELISA performance, we cloned and expressed the high-affinity $4 \mathrm{~m} 5.3$ anti-fluorescein antibody $\left(K_{D}=270 \mathrm{fM}\right)^{18}$ in scFv or in IgG format. Figure 1A shows a schematic illustration of the small molecule-ELISA procedure in different molecular implementations. The cloning strategy and expression vectors for the $4 \mathrm{~m} 5.3$ in scFv and IgG format are shown in Figure 1B and in Supplementary Figures S4-S5. The antibody preparations were pure in size-exclusion chromatography analysis and exhibited peaks of the expected mass in mass spectrometry [Figure 1C and Figure 1D].

In order to confirm the ability of the $4 \mathrm{~m} 5.3$ antibody to stably bind to fluorescein in both $\mathrm{scFv}$ and IgG format, we immobilized the antibody preparations in a BIAcore system and flowed a fluorescein derivative (NH2) onto the microsensor chip. For both antibody preparations, the rapid formation of a kinetically-stable complex was detected [Figure 2].

We performed ELISA experiments using biotinylated CAII preparations, immobilized on streptavidin-coated microtiter plates. Both IgG and $\mathrm{scFv}$ primary reagents allowed the detection of binding events for the small molecule fluoresceinated ligands to CAII [Figure $\mathbf{3 A}$. As expected, the homobivalent and avid interaction

A
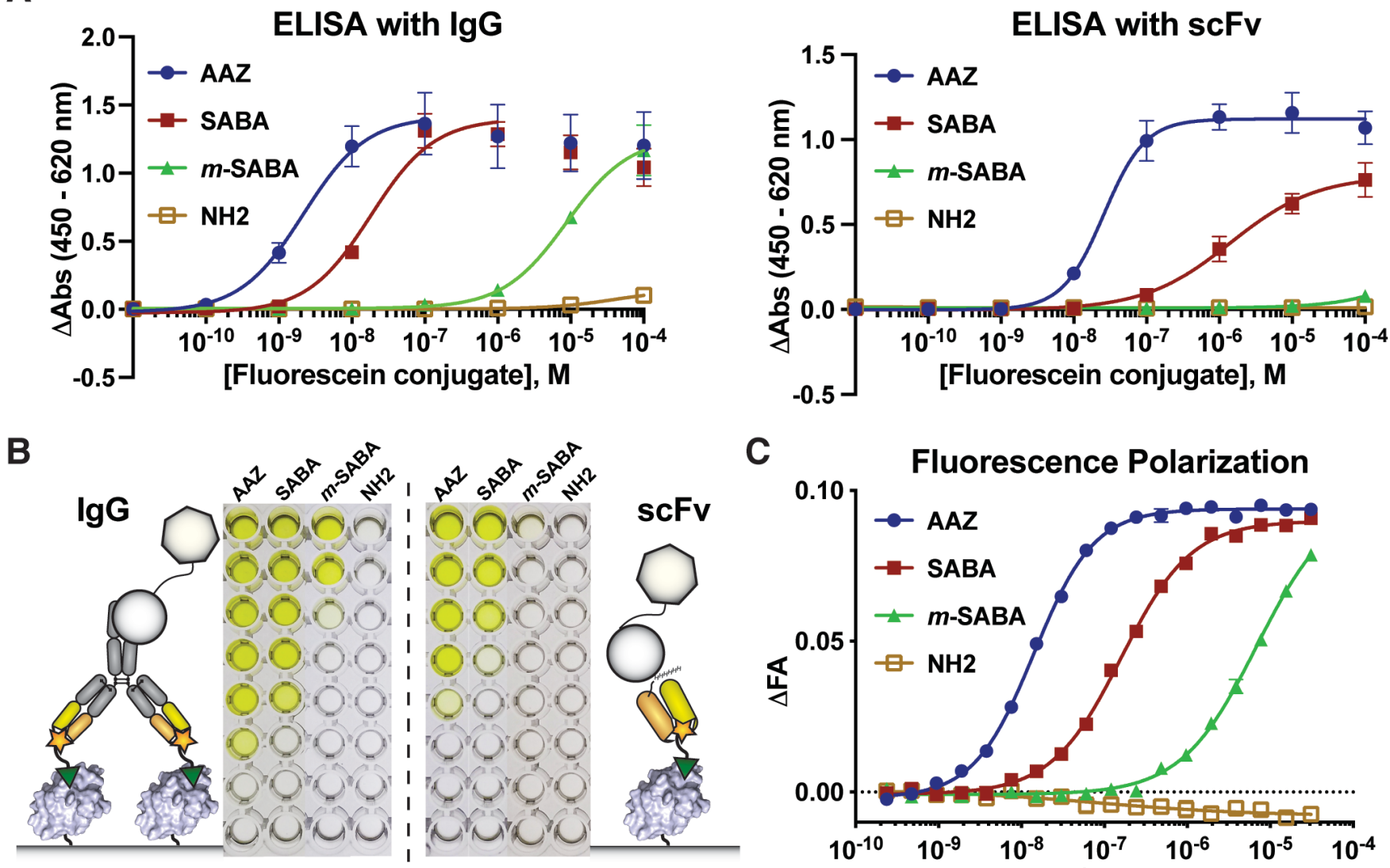

C

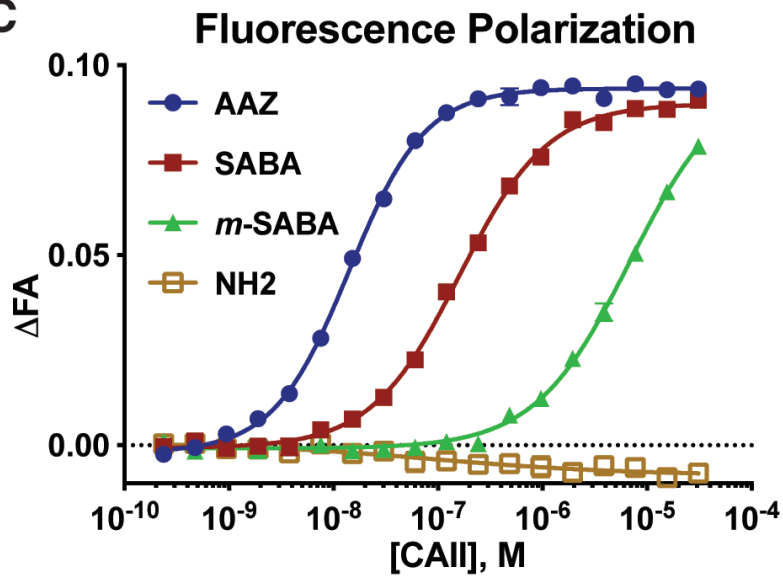

Figure 3. Evaluation of binding affinities of fluoresceinated small molecule ligands against CAII. A) Plastic microtiter plates coated with biotinylated CAII were exposed to a serial dilution of fluorescein-conjugates. In two different experiments, IgG and scFv were used as primary antibody (experiments performed in triplicates) B) Schematic representation and pictures of the small molecule ELISA experiments. C) Fluorescence polarization values of fluorescein-conjugated ligands in presence of a serial dilution of CAII. 
Table 1. Comparison of saturation midpoints data obtained with fluorescence polarization (FP) and Enzyme-Linked ImmunoSorbent Assay (ELISA) of fluoresceinated small molecule ligands of CAII

$\begin{array}{cccc} & \text { FP } & \text { ELISA (IgG) } & \text { ELISA (scFv) } \\ \text { AAZ }^{*} & 14.2 \pm 0.4 \mathrm{nM} & 2.2 \pm 0.3 \mathrm{nM} & 26 \pm 3 \mathrm{nM} \\ \mathrm{SABA} & 160 \pm 7 \mathrm{nM} & 18 \pm 6 \mathrm{nM} & 1.4 \pm 0.2 \mu \mathrm{M} \\ \mathrm{m} \text {-SABA } & 6.8 \pm 0.8 \mu \mathrm{M} & 8.8 \pm 0.3 \mu \mathrm{N} & \text { NA } \\ \mathrm{NH} 2 & \mathrm{NA} & \mathrm{NA} & \mathrm{NA}\end{array}$

*Fluorescein- $\mathrm{PEG}_{2}$-linked aromatic sulfonamides; $\mathrm{AAZ}=$ acetazolamide; $\mathrm{SABA}=4$-sulfamoylbenzoic amide; $m$-SABA = 3-sulfamoylbenzoic amide; $\mathrm{NH} 2$ = free-amino group

provided by the anti-fluorescein IgG resulted in ELISA signals at lower ligand concentrations, compared to the use of scFv detection. In both systems, ELISA signals were highly specific, as evidenced by the very low absorbance detected for the free-amino fluoresceinconjugate (NH2). In solution fluorescence polarization (FP) measurements with the same fluorescein-conjugated ligands were also employed as comparative technique [Figure $3 \mathrm{C}$ and Table $\mathbf{1}$ ].

The monovalent $\mathrm{scFv}$ antibody was also investigated in complex with fluorescein conjugates for the improvement of BIAcore readouts. Figure 4 reports the binding interaction of fluoresceinconjugated ligands with a CAII coated CM5 microsensor chip. Ligand complexation with the $\mathrm{scFv}$ antibody yields to $>6$-fold signal enhancement while the negative control conjugate did not show any binding signal [Table 2]. ELISA experiments were also extended to ligands displayed on nucleic acid scaffolds. For this purpose, CAII ligands conjugated at the 5'-end of a C6-amino linker 12-mer DNA strand were annealed to complementary 12 -mer locked nucleic acid (LNA) strand bearing a fluorescein moiety at the 5'-end [Figure 5]. In a first setting, a serial dilution of the DNA:LNA heteroduplex was detected after incubation on bCAII-coated StreptaWells [Figure 5A]. Whereas DNA structures linked to AAZ and SABA gave high specific signal, $m$-SABA conjugates were not detected in the studied range of concentrations. In order to reduce the consumption of DNA-linked compounds and explore binding events at higher
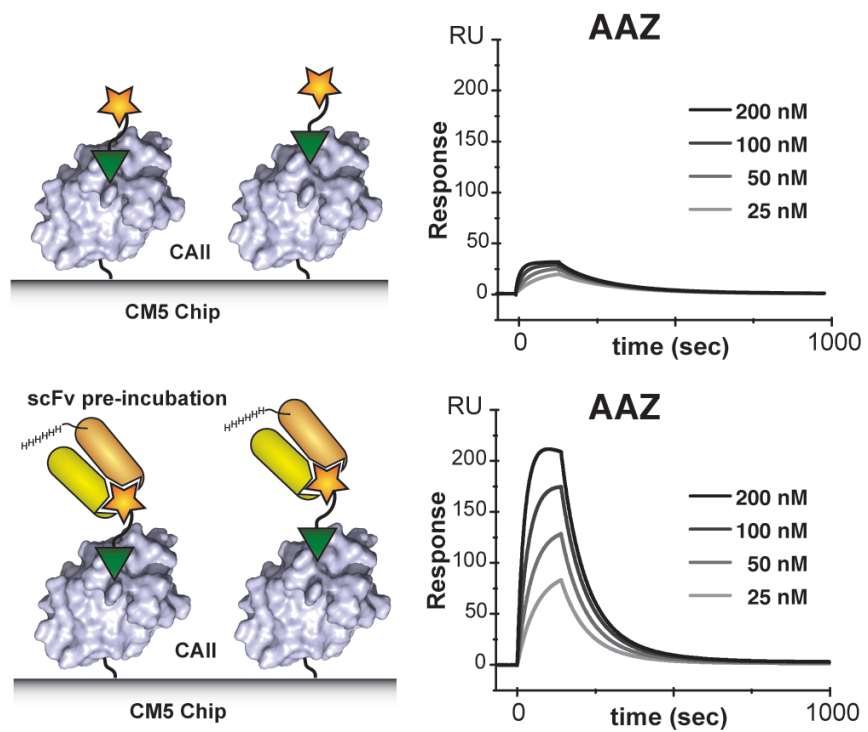
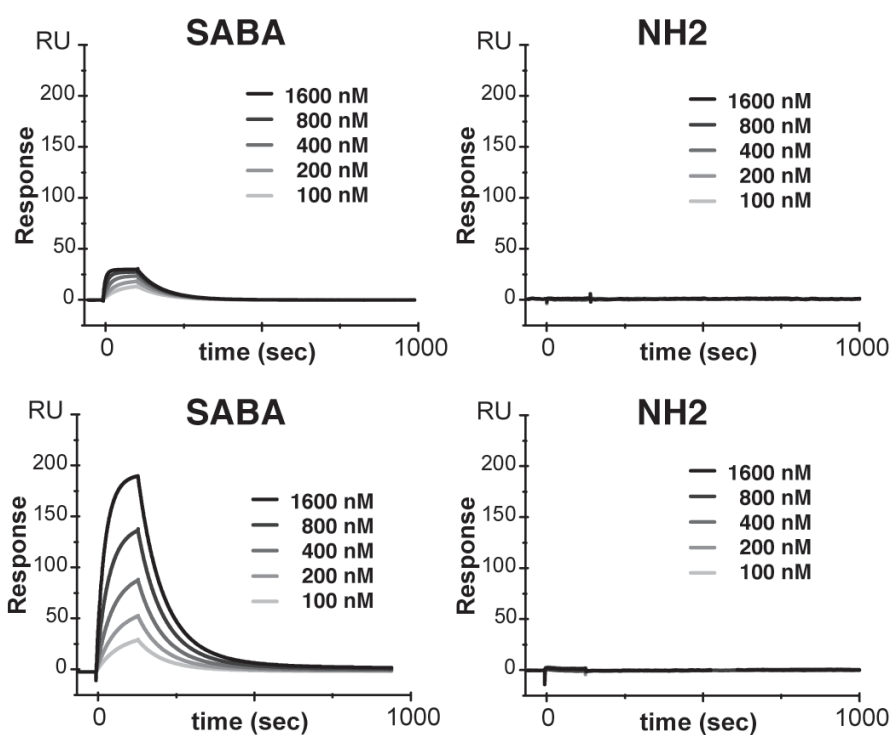

Figure 4. BIAcore sensograms of fluorescein-conjugated ligands (i.e., AAZ, SABA and NH2) on covalently modified CM5 Series $S$ sensor chip with CAII (immobilized density 1145 RU). Free-ligands (top-row) or pre-formed complex with the scFv (bottom-row) were analyzed at different concentrations.

Table 2. Fitted kinetics data of binding interactions of fluoresceinated small molecule ligands on CAII coated CM5 BIAcore chip.

\begin{tabular}{|c|c|c|c|c|c|c|c|c|}
\hline & \multicolumn{4}{|c|}{ Free-ligands } & \multicolumn{4}{|c|}{ scFv complex } \\
\hline & $\begin{array}{c}\mathrm{k}_{\mathrm{on}} \\
\left(\cdot 10^{4} \mathrm{M}^{-1} \mathrm{~s}^{-1}\right)\end{array}$ & $\begin{array}{c}k_{\text {off }} \\
\left(\cdot 10^{-3} \mathrm{~s}^{-1}\right)\end{array}$ & $\begin{array}{l}\mathrm{K}_{\mathrm{D}} \\
(\mathrm{nM})\end{array}$ & $\mathrm{R}_{\max }$ & $\begin{array}{c}k_{\text {on }} \\
\left(\cdot 10^{4} \mathrm{M}^{-1} \mathrm{~s}^{-1}\right)\end{array}$ & $\begin{array}{c}k_{\text {off }} \\
\left(\cdot 10^{-3} \mathrm{~s}^{-1}\right)\end{array}$ & $\begin{array}{c}\mathrm{K}_{\mathrm{D}} \\
(\mathrm{nM})\end{array}$ & $\mathrm{R}_{\max }$ \\
\hline AAZ & $55.490 \pm 0.002$ & $7.94 \pm 0.02$ & 14.3 & 34 & $21.650 \pm 0.002$ & $10.190 \pm 0.004$ & 47.1 & 251 \\
\hline SABA & $8.070 \pm 0.004$ & $10.860 \pm 0.005$ & 135 & 33 & $1.490 \pm 0.001$ & $9.779 \pm 0.004$ & 656 & 252 \\
\hline
\end{tabular}

SPR sensograms were evaluated with BIAcore S200 evaluation software (version 1.0) using 1:1 binding interaction fit. 
A
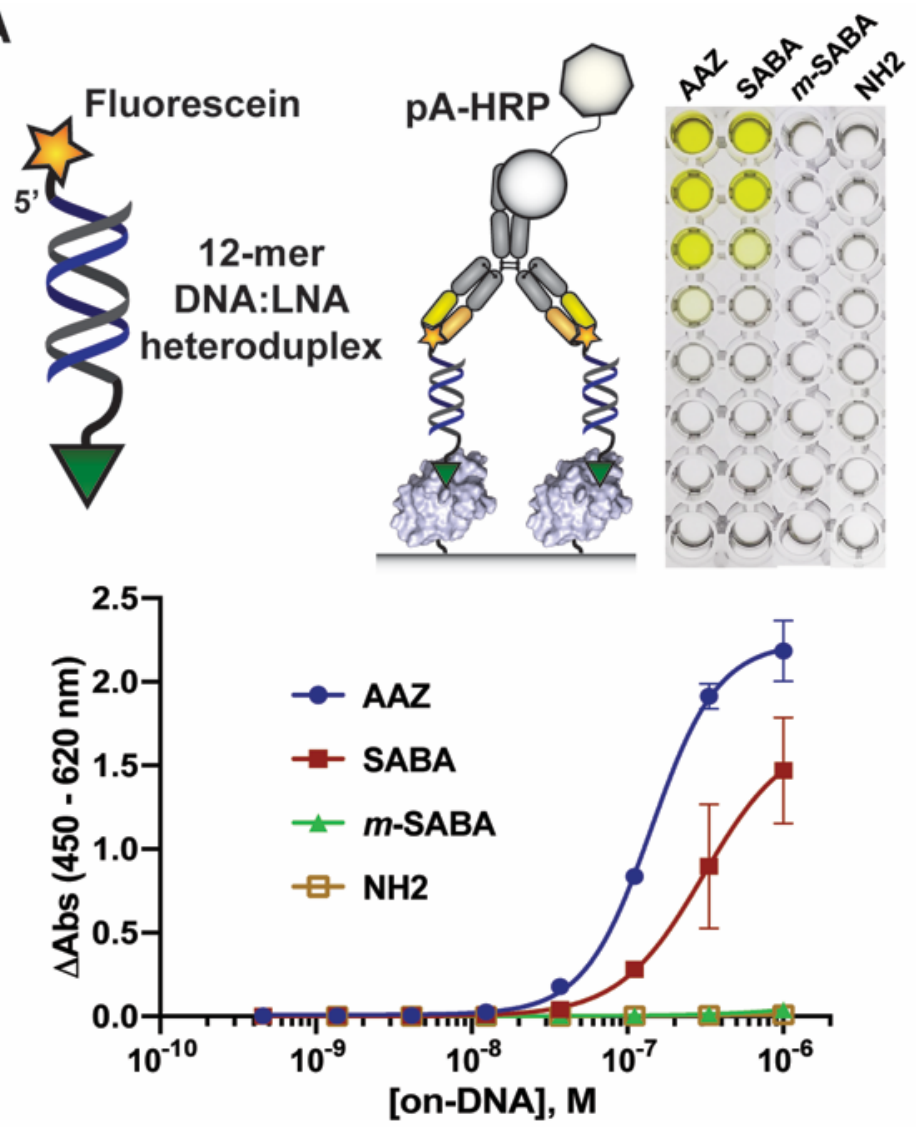

B
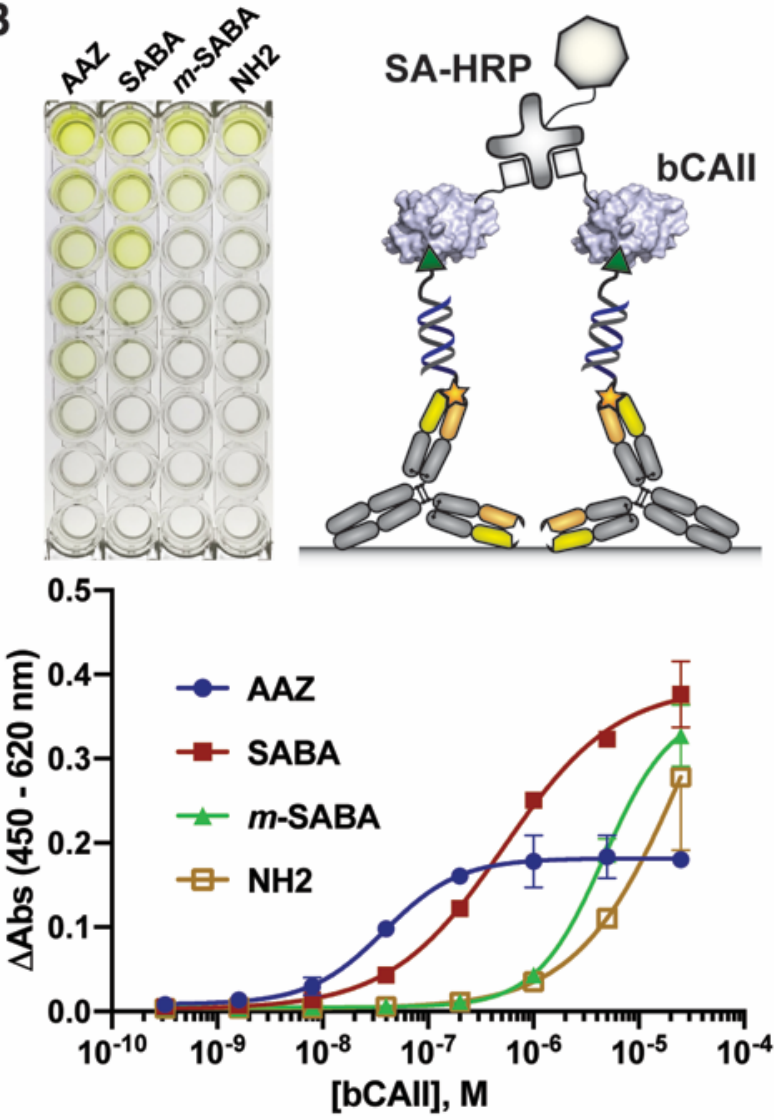

Figure 5. Affinities of CAII ligands displayed on a fluoresceinated DNA:LNA heteroduplex scaffold were measured in two different ELISA experiments. A) A serial dilution of DNA-conjugates was incubated on StreptaWells coated with biotinylated CAII (bCAII). Color development was detected through incubation steps with IgG and horseradish peroxidase conjugated to protein A (pA-HRP). B) 10 nM fluoresceinated DNA-conjugates were immobilized on IgG pre-coated Nunc MaxiSorp ${ }^{\mathrm{TM}}$ plates. A serial dilution of bCAII was detected through incubation with streptavidin-HRP protein conjugate (SA-HRP).

Table 3. Comparison of saturation midpoints data of fluoresceinated DNA-conjugates obtained with ELISA

$\begin{array}{ccc} & \text { DNA titration } & \text { bCAII titration } \\ \text { AAZ } & 142 \pm 3 \mathrm{nM} & 38 \pm 1 \mathrm{nM} \\ \text { SABA } & 307 \pm 7 \mathrm{nM} & 519 \pm 58 \mathrm{nM} \\ m \text {-SABA } & \text { NA } & 4.67 \pm 0.09 \mu \mathrm{M} \\ \mathrm{NH} 2 & \mathrm{NA} & \mathrm{NA}\end{array}$

bCAII = biotinylated carbonic anhydrase II

concentrations, an inverse-ELISA strategy was employed [Figure 5B]. IgG coated Nunc MaxiSorp ${ }^{\mathrm{TM}}$ ELISA stripes were enriched with fluoresceinated DNA-structures at low concentration (i.e., 10 $\mathrm{nM}$ ) and exposed to a serial dilution of biotinylated CAII. Binding interactions were detected upon incubation with streptavidin-HRP conjugate yielding to highly specific signals [Table 3].

\section{DISCUSSION}

Two variants ( $\mathrm{scFv}$ and $\mathrm{IgG}$ ) of a high-affinity antibody directed against fluorescein were investigated for the detection of protein binding properties of fluoresceinated small molecule conjugates. The experimental procedures were successfully implemented in both ELISA and BIAcore methodologies, alleviating technical limitations which are often encountered using small molecule ligands.
Carbonic anhydrase 2 represented an ideal biochemical model since binders with dissociation constants ranging from nanomolar to single-digit micromolar were available.

The small molecule ELISA methodology exhibited a performance similar to the one obtained with monoclonal antibodies, which are routinely used for research purposes and for clinical chemistry applications. ${ }^{25}$ The technology features a very low consumption of reagents thus lending itself to high-throughput screening. Complexation of fluoresceinated ligands with the $4 \mathrm{~m} 5.3$ antibody in IgG format allowed the formation of stable CAII complexes, which facilitated the detection of low-affinity binding interactions (e.g., $m$ SABA) due to an avidity effect. ELISA measurements performed in the presence of the monovalent $\mathrm{scFv}$ antibody prevented multivalent interactions and yielded dissociation constant in excellent agreement with fluorescence polarization data [Table 1 ].

The formation of stable 1:1 complexes between individual fluorescein-conjugates and $\mathrm{scFv}(4 \mathrm{~m} 5.3)$ proved to be particularly useful in the context of BIAcore experiments. The characterization of small molecule ligands using surface plasmon resonance is notoriously problematic, as it may suffer from solubility limitations and from insufficient signal intensity (BIAcore signals are proportional to the mass on the microsensor chip). Ligand complexation with $\mathrm{scFv}(4 \mathrm{~m} 5.3)$ increased the total molecular weight of fluoresceinated ligands and substantially increased the resonance units and the quality of sensograms [Figure 4]. Complex formation with the antibody alleviates the need for the use of organic solvents and allow to coat 
protein targets at a lower surface density. The extremely high stability of the scFv:fluorescein complex [Figure 2] allowed the execution of experiments down to the nanomolar concentration range, without altering kinetic and thermodynamic binding parameters compared to solution measurements by FP [Table 2].

We extended the small molecule ELISA procedure and applied in the context of small molecule-DNA conjugates. The newly described methods may be broadly applicable in view of the growing popularity of DNA-Encoded Libraries as a powerful tools for ligand discovery. ${ }^{13,26}$ Using DEL technology, one often needs to validate hundreds of hit-compounds resulting from affinity-capture selections, decoded by high-throughput DNA sequencing. The covalent linking of small-molecules on-DNA increases solubility and allows a low consumption of reagents. Moreover, it is often convenient to synthesize, purify and characterize (e.g., by mass spectrometry) the small molecule conjugates on DNA, using the same procedures as for library synthesis. Heteroduplex formation could also be performed using fluoresceinated locked nucleic acids (LNA), which can be shorter compared to DNA because of an increased stability. Fluoresceinated DNA-structures can be coated in minute amount on ELISA plates and detected using a serial dilution of the taggedprotein of interest [Figure 5B].

\section{CONCLUSIONS}

The discovery of small molecule pharmaceuticals crucially relies on the confident identification of protein binders. ${ }^{27}$ Ligand discovery is also important for the Chemical Biology characterization of biological processes ${ }^{28-30}$ and for the development of innovative affinity chromatography supports. ${ }^{31,32}$ Small molecule targeting agents can be used for pharmacodelivery applications (e.g., small moleculedrug conjugates, universal CAR T-cells) and for the selective degradation of target proteins using PROTAC technology. ${ }^{33-35}$

We used fluoresceinated ligands and high-affinity anti-fluorescein antibodies in order to adapt ELISA and BIAcore methodologies to the world of small molecules. The methods described in this article represent an inexpensive approach for the study of protein recognition events, which can deliver both qualitative and quantitative information (e.g., $\mathrm{K}_{\mathrm{D}}$ dissociation constants). Complex formation with antibodies increases ligand solubility and enhances signal detection, both in ELISA and in BIAcore. Our findings are particularly useful in the context of DEL technology, where libraries of unprecedented size may deliver hundreds of putative hit compounds per round of affinity selection. ${ }^{14}$ Facile resynthesis and hit validation procedures, which can be performed on-DNA, may contribute to an even greater acceptance of DEL technology both in Industry and in Academia. $^{12}$

\section{ASSOCIATED CONTENT}

\section{Supporting Information}

The Supporting Information is available free of charge on the ACS Publications website.

Supplementary Information (PDF)

\section{AUTHOR INFORMATION}

\section{Corresponding Author}

* Dario Neri - Institute of Pharmaceutical Sciences, ETH Zürich, 8093 Zürich, Switzerland; Email: dario.neri@pharma.ethz.ch

\section{Author Contributions}

M.C. and D.N. wrote the manuscript. M.C. cloned and expressed antibodies. M.C. performed ELISA. M.C. and S.O. performed SPR measurements. L.P. synthesized fluorescein-linked compounds. All authors have given approval to the final version of the manuscript.

\section{Notes}

The authors declare the following competing financial interest(s): D.N. is a cofounder and shareholder of Philochem AG.

\section{ACKNOWLEDGMENT}

This project has received funding from the European Research Council (ERC) under the European Union's Horizon 2020 research and innovation program (grant agreement 670603) and from the Swiss National Science Foundation (Grant Nr. 310030B_182003/1). We cordially thank Dr. Cyrill Brunner (ETH Zürich) for fruitful discussions and Martina Bigatti (Philochem AG) for the help with the DNAlinked compounds.

\section{ABBREVIATIONS}

ELISA, Enzyme-Linked ImmunoSorbent Assay; SPR, Surface Plasmon Resonance, FP, Fluorescence Polarization; CAII, Carbonic anhydrase 2; AAZ, acetazolamide; SABA, 4-sulfamoylbenzoic amide; $m$ $\mathrm{SABA}$, 3-sulfamoylbenzoic amide; IgG, Immunoglobulin; scFv, single-chain variable fragment; DEL, DNA-Encoded Library.

\section{REFERENCES}

(1) Renaud, J.-P.; Chung, C.; Danielson, U. H.; Egner, U.; Hennig, M.; Hubbard, R. E.; Nar, H. Biophysics in Drug Discovery: Impact, Challenges and Opportunities. Nat. Rev. Drug Discov. 2016, 15 (10), 679-698

(2) Baell, J.; Walters, M. A. Chemistry: Chemical Con Artists Foil Drug Discovery. Nature 2014, 513 (7519), 481-483.

(3) Nero, T. L.; Morton, C. J.; Holien, J. K.; Wielens, J.; Parker, M. W. Oncogenic Protein Interfaces: Small Molecules, Big Challenges. Nat. Rev. Cancer 2014, 14 (4), 248-262.

(4) Scott, D. E.; Bayly, A. R.; Abell, C.; Skidmore, J. Small Molecules, Big Targets: Drug Discovery Faces the Protein-Protein Interaction Challenge. Nat. Rev. Drug Discov. 2016, 15 (8), 533-550.

(5) Santos, R.; Ursu, O.; Gaulton, A.; Bento, A. P.; Donadi, R. S.; Bologa, C. G.; Karlsson, A.; Al-Lazikani, B.; Hersey, A.; Oprea, T. I.; Overington, J. P. A Comprehensive Map of Molecular Drug Targets. Nat. Rev. Drug Discov. 2017, 16 (1), 19-34.

(6) Makley, L. N.; Gestwicki, J. E. Expanding the Number of 'Druggable' Targets: Non-Enzymes and Protein-Protein Interactions. Chem. Biol. Drug Des. 2013, 81 (1), 22-32.

(7) Falconer, R. J. Applications of Isothermal Titration Calorimetry the Research and Technical Developments from 2011 to 2015. J. Mol. Recognit. 2016, 29 (10), 504-515.

Pellecchia, M.; Bertini, I.; Cowburn, D.; Dalvit, C.; Giralt, E.; Jahnke, W.; James, T. L.; Homans, S. W.; Kessler, H.; Luchinat, C.; Meyer, B.; Oschkinat, H.; Peng, J.; Schwalbe, H.; Siegal, G. Perspectives on NMR in Drug Discovery: A Technique Comes of Age. Nat. Rev. Drug Discov. 2008, 7(9), 738-745.

Drescher, D. G.; Selvakumar, D.; Drescher, M. J. Analysis of Protein Interactions by Surface Plasmon Resonance; 2018; pp 1-30.

(10) Patching, S. G. Surface Plasmon Resonance Spectroscopy for Characterisation of Membrane Protein-Ligand Interactions and Its Potential for Drug Discovery. Biochim. Biophys. Acta - Biomembr. 2014, 1838 (1), 43-55.

Myszka, D. G.; Rich, R. L. Implementing Surface Plasmon Resonance Biosensors in Drug Discovery. Pharm. Sci. Technolo. Today 2000, 3(9), 310-317.
Neri, D.; Lerner, R. A. DNA-Encoded Chemical Libraries: A Selection System Based on Endowing Organic Compounds with Amplifiable Information. Annu Rev Biochem 2018, 87, 479-502. Goodnow, R. A.; Dumelin, C. E.; Keefe, A. D. DNA-Encoded 
Chemistry: Enabling the Deeper Sampling of Chemical Space. Nat. Rev. Drug Discov. 2017, 16 (2), 131-147.

(14) Favalli, N.; Bassi, G.; Scheuermann, J.; Neri, D. DNA-Encoded Chemical Libraries - Achievements and Remaining Challenges. FEBS Lett. 2018, 592 (12), 2168-2180.

(15) Yalow, R. S.; Berson, S. A. IMMUNOASSAY OF ENDOGENOUS PLASMA INSULIN IN MAN. J. Clin. Invest. 1960, 39 (7), 11571175.

(16) Lequin, R. M. Enzyme Immunoassay (EIA)/Enzyme-Linked Immunosorbent Assay (ELISA). Clin. Chem. 2005, 51 (12), 24152418.

(17) Midelfort, K. S.; Hernandez, H. H.; Lippow, S. M.; Tidor, B.; Drennan, C. L.; Wittrup, K. D. Substantial Energetic Improvement with Minimal Structural Perturbation in a High Affinity Mutant Antibody. J. Mol. Biol. 2004, 343 (3), 685-701.

(18) Boder, E. T.; Midelfort, K. S.; Wittrup, K. D. Directed Evolution of Antibody Fragments with Monovalent Femtomolar AntigenBinding Affinity. Proc. Natl. Acad. Sci. 2000, 97 (20), 1070110705 .

(19) Borsi, L.; Balza, E.; Bestagno, M.; Castellani, P.; Carnemolla, B.; Biro, A.; Leprini, A.; Sepulveda, J.; Burrone, O.; Neri, D.; Zardi, L. Selective Targeting of Tumoral Vasculature: Comparison of Different Formats of an Antibody (L19) to the ED-B Domain of Fibronectin. Int. J. Cancer 2002, 102 (1), 75-85.

(20) Rajendra, Y.; Kiseljak, D.; Baldi, L.; Hacker, D. L.; Wurm, F. M. A Simple High-Yielding Process for Transient Gene Expression in CHO Cells. J. Biotechnol. 2011, 153 (1-2), 22-26.

(21) Sannino, A.; Gironda-Martínez, A.; Gorre, É. M. D.; Prati, L.; Piazzi, J.; Scheuermann, J.; Neri, D.; Donckele, E. J.; Samain, F. Critical Evaluation of Photo-Cross-Linking Parameters for the Implementation of Efficient DNA-Encoded Chemical Library Selections. ACS Comb. Sci. 2020, acscombsci.0c00023.

(22) Zimmermann, G.; Li, Y.; Rieder, U.; Mattarella, M.; Neri, D.; Scheuermann, J. Hit-Validation Methodologies for Ligands Isolated from DNA-Encoded Chemical Libraries. Chembiochem 2017, 18 (9), 853-857.

(23) Swain, B.; Angeli, A.; Angapelly, S.; Thacker, P. S.; Singh, P.; Supuran, C. T.; Arifuddin, M. Synthesis of a New Series of 3Functionalised-1-Phenyl-1,2,3-Triazole Sulfamoylbenzamides as Carbonic Anhydrase I, II, IV and IX Inhibitors. J. Enzyme Inhib. Med. Chem. 2019, 34(1), 1199-1209.

(24) Poulsen, S.-A.; Bornaghi, L. F.; Healy, P. C. Synthesis and Structure-Activity Relationships of Novel Benzene Sulfonamides with Potent Binding Affinity for Bovine Carbonic Anhydrase II. Bioorg. Med. Chem. Lett. 2005, 15 (24), 5429-5433.
Gan, S. D.; Patel, K. R. Enzyme Immunoassay and Enzyme-Linked Immunosorbent Assay. J. Invest. Dermatol. 2013, 133 (9), 1-3.

Ottl, J.; Leder, L.; Schaefer, J. V; Dumelin, C. E. Encoded Library Technologies as Integrated Lead Finding Platforms for Drug Discovery. Molecules 2019, 24 (8).

(27) Moffat, J. G.; Rudolph, J.; Bailey, D. Phenotypic Screening in Cancer Drug Discovery - Past, Present and Future. Nat. Rev. Drug Discov. 2014, 13 (8), 588-602.

(28) Arrowsmith, C. H.; Audia, J. E.; Austin, C.; Baell, J.; Bennett, J.; Blagg, J.; Bountra, C.; Brennan, P. E.; Brown, P. J.; Bunnage, M. E.; Buser-Doepner, C.; Campbell, R. M.; Carter, A. J.; Cohen, P.; Copeland, R. A.; Cravatt, B.; Dahlin, J. L.; Dhanak, D.; Edwards, A. M.; Frederiksen, M.; Frye, S. V; Gray, N.; Grimshaw, C. E.; Hepworth, D.; Howe, T.; Huber, K. V; Jin, J.; Knapp, S.; Kotz, J. D.; Kruger, R. G.; Lowe, D.; Mader, M. M.; Marsden, B.; MuellerFahrnow, A.; Muller, S.; O’Hagan, R. C.; Overington, J. P.; Owen, D. R.; Rosenberg, S. H.; Roth, B.; Ross, R.; Schapira, M.; Schreiber, S. L.; Shoichet, B.; Sundstrom, M.; Superti-Furga, G.; Taunton, J.; Toledo-Sherman, L.; Walpole, C.; Walters, M. A.; Willson, T. M.; Workman, P.; Young, R. N.; Zuercher, W. J. The Promise and Peril of Chemical Probes. Nat Chem Biol2015, 11 (8), 536-541.

(29) Blagg, J.; Workman, P. Choose and Use Your Chemical Probe Wisely to Explore Cancer Biology. Cancer Cell 2017, 32 (1), 9-25.

(30) Lukinavičius, G.; Reymond, L.; Umezawa, K.; Sallin, O.; D’Este, E.; Göttfert, F.; Ta, H.; Hell, S. W.; Urano, Y.; Johnsson, K. Fluorogenic Probes for Multicolor Imaging in Living Cells. J. Am. Chem. Soc. 2016, 138 (30), 9365-9368.

(31) Ursu, A.; Waldmann, H. Hide and Seek: Identification and Confirmation of Small Molecule Protein Targets. Bioorg. Med. Chem. Lett. 2015, 25 (16), 3079-3086.

(32) Alves, N. J.; Stimple, S. D.; Handlogten, M. W.; Ashley, J. D.; Kiziltepe, T.; Bilgicer, B. Small-Molecule-Based Affinity Chromatography Method for Antibody Purification via Nucleotide Binding Site Targeting. Anal. Chem. 2012, 84 (18), 7721-7728.

(33) Zhuang, C.; Guan, X.; Ma, H.; Cong, H.; Zhang, W.; Miao, Z. Small Molecule-Drug Conjugates: A Novel Strategy for Cancer-Targeted Treatment. Eur.J. Med. Chem. 2019, 163, 883-895.

(34) Paiva, S.-L.; Crews, C. M. Targeted Protein Degradation: Elements of PROTAC Design. Curr. Opin. Chem. Biol. 2019, 50, 111-119.

(35) Lee, Y. G.; Marks, I.; Srinivasarao, M.; Kanduluru, A. K. Mahalingam, S. M.; Liu, X.; Chu, H.; Low, P. S. Use of a Single CAR $\mathrm{T}$ Cell and Several Bispecific Adapters Facilitates Eradication of Multiple Antigenically Different Solid Tumors. Cancer Res. 2019, 79(2), 387-396 
TOC

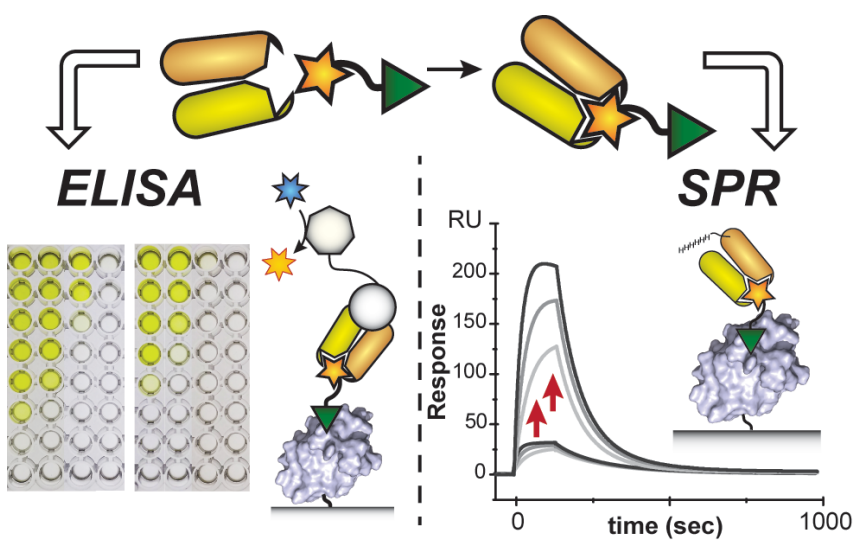

